\title{
Partizipative Wissenserzeugung und Wissenschaftlichkeit - ein methodologischer Beitrag
}

\author{
Rico Defila \& Antonietta Di Giulio
}

\section{Die Belastbarkeit partizipativ gewonnenen Wissens - ein Thema (auch) für transdisziplinäre Forschung außerhalb und innerhalb von Reallaborforschung}

Den Referenzpunkt dieses Beitrags bilden Forschungsprojekte, an denen sowohl Forscherinnen und Forscher als auch Personen aus der Praxis beteiligt sind. Solche Projekte sind nicht in erster Linie auf eine wissenschaftsbasierte Planung oder Entwicklung ausgerichtet oder darauf, bereits bekannte Erkenntnisse und Lösungen umzusetzen, sondern (mindestens: auch) darauf, neues Wissen zu produzieren. Sie verfolgen also (mindestens: auch) das Ziel, belastbare Einsichten zu produzieren, die innerhalb der Wissenschaft aufgegriffen werden können. Damit sollen sie einen Beitrag leisten ,zur Weiterentwicklung wissenschaftlichen Wissens" (Altrichter und Feindt 2008, S. 461). Um diesen Zweck erfüllen zu können, muss das erzeugte Wissen einer wissenschaftlichen Qualitätsprüfung unterzogen werden können und standhalten, d. h., es muss sich ,in einem wissenschaftlichen Diskurs bewähren“ (ebd.). Innerhalb der Menge an Projekten, die die genannten Merkmale aufweisen, fokussiert der vorliegende Beitrag auf Projekte, die der transdisziplinären Forschung und der Reallabor-Forschung zugeordnet werden können.

Transdisziplinäre Forschung wird hier akteurorientiert verstanden ${ }^{1}$ als Variante einer auf eine Synthese ausgerichteten interdisziplinären Forschung. Ein Forschungsprozess ist also dann transdisziplinär, wenn sich daran sowohl Forscherinnen und Forscher aus verschiedenen wissenschaftlichen Disziplinen beteiligen

1 Zur Abgrenzung dieses Verständnisses von einem eher wissenschaftstheoretisch gefärbten Verständnis s. z. B. Klein $(2010 ; 2014)$ oder auch bereits Defila und Di Giulio (1998). 
(sogenannte „certified experts“), als auch Praxisakteure (sogenannte „,non-certified experts"). Dieses Verständnis geht also von Expertise als Kriterium für die Beteiligung an der Produktion neuen Wissens aus und nicht von Kriterien wie Mitsprache-Recht, gesellschaftliche Rolle, Rolle im Praxisfeld oder Betroffenheit (s. z. B. Collins und Evans 2002; Defila und Di Giulio 2015). Praxisakteure wiederum sind Akteure, an die sich die Ergebnisse der Forschung richten (Anwenderinnen und Anwender) und die bezogen auf das untersuchte Thema über eine Praxis-Expertise verfügen, die die Forschungs-Expertise der Forscherinnen und Forscher ergänzt. ${ }^{2}$ Das können Angehörige von Berufsfeldern bzw. Praxisfeldern, gesellschaftlichen Gruppen, zivilgesellschaftlichen Organisationen (Verein, Verband etc.), privatwirtschaftlichen Unternehmen oder öffentlich-rechtlichen Einrichtungen (Ministerium, Verwaltungseinheit, Bildungseinrichtung etc.) sein. Solche Akteure können als gleichberechtigte Mitglieder eines Projektteams einbezogen werden oder als externe Beteiligte, die substantiell am Projekt beteiligt, aber nicht Projektteam-Mitglieder sind (zu diesen Unterscheidungen s. Defila et al. 2006, S. 216f.). Sind Praxisakteure lediglich Untersuchungsgegenstand oder Zielpublikum der Ergebnisse eines Projekts, oder sind sie lediglich ,Echoraum' für die Forschenden, handelt es sich gemäß diesem Verständnis nicht um eine transdisziplinäre Zusammenarbeit (ebd.). In den Worten von Mobjörk (2010) ist das hier zugrunde gelegte Verständnis von Transdisziplinarität eine ,participatory transdisciplinarity“ im Unterschied zu einer „,consulting transdisciplinarity“.

Zur Frage, was Reallabore sind und was Reallaborforschung kennzeichnet, ist die Debatte gerade erst gestartet. Diese Debatte mit allen offenen Fragen soll an dieser Stelle nicht reproduziert werden. Vielmehr soll dafür auf Arnold und Piontek (2018) sowie auf Beecroft et al. (2018) verwiesen werden (s. aber z. B. auch GAIA Special Issue 2018; Schäpke et al. 2017; TATuP-Schwerpunkt 2016; BGLThemenheft 2017; Wagner und Grunwald 2015). Für den vorliegenden Beitrag von Bedeutung sind drei Merkmale von Reallabor-Projekten, die in dieser Allgemeinheit unbestritten sein dürften:

- Reallabore wollen neues Wissen erzeugen, sie verfolgen also erkenntnisbezogene Ziele (Forschungsziele).

- Reallabore verfolgen explizit auch transformative Ziele (Praxisziele). Gegenstand der laufenden Diskussion ist die relative Gewichtung von erkenntnisbezogenen Zielen und transformativen Zielen.

- Reallaborprojekte sind partizipativ auf die Beteiligung von Praxisakteuren angelegt. Gegenstand der laufenden Diskussion ist hier, inwieweit solche Akteure aufgrund ihrer Expertise beteiligt werden sollen oder aufgrund anderer

2 Je nach untersuchtem Thema können auch Wissenschaftlerinnen und Wissenschaftler Praxisakteure in einem transdisziplinären Projekt sein. 
Kriterien, wie z. B. Rolle bezogen auf eine bestimmte soziale Innovation, Betroffenheit oder Mitsprache-Recht, resp. inwieweit sich aus unterschiedlichen Kriterien unterschiedliche Formen der Partizipation in ein und demselben Reallabor ergeben (können), je nachdem, ob die Partizipation jeweils einem Forschungsziel oder einem Praxisziel dient (s. dazu auch Beecroft et al. 2018 sowie Eckart et al. 2018).

Reallabore und transdisziplinäre Projekte haben also zwei Gemeinsamkeiten, die partizipative Anlage und den Anspruch, neues Wissen zu produzieren. ${ }^{3}$ Der vorliegende Beitrag ist dem Zusammenhang zwischen Partizipation und der Produktion neuen Wissens gewidmet, wobei auf die wissenschaftliche Belastbarkeit der erzeugten Erkenntnisse, d. h. auf deren Wissenschaftlichkeit, fokussiert wird. Bewusst ausgeklammert wird hier also die Frage nach der Legitimität transformativer Ziele und der diesbezüglichen Bedeutung von Partizipation (diese Frage ist sehr wichtig, sie stellt aber eine eigene und getrennt zu führende Debatte dar, s. dazu auch Defila und Di Giulio 2018 bzw. Eckart et al. 2018).

Forschende, die partizipativ forschen, sind mit Blick auf die Akzeptanz ihrer Vorgehensweisen und Ergebnisse innerhalb der Wissenschaft oft damit konfrontiert, dass ihnen Skepsis entgegengebracht wird bzw. dass sie nicht rezipiert werden. Zur Erklärung der fehlenden Anerkennung benennen Forscherinnen und Forscher unterschiedliche Ursachen. Einige führen sie zurück auf die „fehlende Selbstverständlichkeit“ in der Anwendung von „partizipativen Strategien“, die wiederum zur Folge habe, dass sich ,schnell skeptische Stimmen [melden], welche die wissenschaftliche Dignität von Forschungsergebnissen infrage stellen, denen auf den ersten Blick die Erkenntnisdistanz zum Alltagswissen fehlt" (Bergold und Thomas 2010, S. 333). Eine weitere Erklärung für die fehlende Anerkennung ist die Dominanz eines „,nomothetischen Forschungsansatzes“ in der Wissenschaft (ebd., S. 342). Andere sehen sie als Ausdruck der „Sorge“, ob „Forschung ihr kritisches und entdeckendes Potenzial entfalten [kann], wenn das Begehren auf bestimmte Handlungsziele gerichtet ist" (Altrichter und Feindt 2008, S. 459). Das betrifft auch Forschende, die außerhalb oder innerhalb von Reallaborprojekten transdisziplinär forschen. So konstatiert etwa Ukowitz, dass unabhängig von den Ursachen „den ForscherInnen die Erfahrung [gemeinsam ist], dass sich transdisziplinäre Forschung nicht reibungslos in die akademische Wissenschaft einfügen lässt" (Ukowitz 2012, S. 78).

Zusammengefasst liegen ausreichend viele Erfahrungen vor, um folgern zu können, dass innerhalb der Wissenschaft Zweifel an der Gültigkeit von partizipativ erzeugtem Wissen bestehen, d. h., dass dessen Wissenschaftlichkeit infrage ge-

3 Transdisziplinäre Forschung wird hier also nicht zwingend als transformative Forschung aufgefasst, im Unterschied zu Ansätzen wie z. B. dem von Krohn, Grunwald und Ukowitz (2017). 
stellt wird. Das wiederum ist ein erster guter Grund, sich mit der Frage nach wissenschaftlichen Gütekriterien für partizipativ erzeugte Erkenntnisse transdisziplinärer Forschung zu befassen und damit auch mit der Frage, wie solchen Zweifeln begegnet werden kann. ${ }^{4}$

\section{Der Kontext der partizipativen Forschung}

Mit Blick auf die Frage nach den wissenschaftlichen Gütekriterien für partizipativ erzeugte Erkenntnisse ist es sinnvoll, sich in einem ersten Schritt zu vergewissern, in welchen Diskursen nach Beiträgen zu dieser Frage gesucht werden kann. Diesem Ziel sind die nachstehenden Ausführungen gewidmet, wobei es im gegebenen Rahmen nicht möglich ist, diese Diskurse ausführlich aufzurollen. Die Forschung in transdisziplinären Projekten und in Reallaboren kann aus der Perspektive, die für diesen Beitrag eingenommen wird, dem weiten Feld partizipativer Forschung zugeschlagen werden: „Nicht Forschung über Menschen und auch nicht für Menschen, sondern Forschung mit Menschen - dies ist der Anspruch und die grundlegende erkenntnistheoretische Position von partizipativer Forschung" (Bergold und Thomas 2010, S. 333).

Partizipative Forschung gehört zur Tradition der so genannten „Aktionsforschung", die in den 1970er Jahren in Deutschland eine Blütezeit hatte (s. z. B. von Unger 2014, S. 13ff.). Im Rahmen der Aktionsforschung „,wurde (...) eine Methodik entwickelt, die über die Wissensproduktion nicht nur Partei ergreift, sondern ebenso für praktische Veränderungen eintritt. Aktionsforschung verstand sich unter dem Anspruch der Demokratisierung und Emanzipation von ungerechten, menschenunwürdigen, repressiven Verhältnissen daher als ,Methode sozialer Veränderung'. Die Verbesserung der Lebensumstände von sozial benachteiligten Bevölkerungsgruppen machte es demzufolge notwendig, diese als aktiv Mitwirkende in den Forschungsprozess einzubeziehen, wohingegen die Forschenden ihre Dominanzrolle aufgeben sollten“ (Bergold und Thomas 2010, S. 333f.; ähnlich auch Altrichter und Feindt 2008). Dazu gehört(e) auch die Durchführung

4 Die Prämisse, von der dieser Beitrag ausgeht, ist, dass das Wissenschaftssystem als ein sich selbst regulierendes und reproduzierendes System, in dem Qualität durch Peers definiert, geprüft und zugeschrieben wird, im Detail Mängel aufweist und in vielen Dingen sicher verbesserungswürdig ist, aber grosso modo funktioniert. Sollen Veränderungen im Wissenschaftssystem angeregt oder sogar herbeigeführt werden, müssen sowohl die Veränderungen selbst als auch deren Herbeiführung auf die Funktionsweise des Systems zugeschnitten sein. Sich mit wissenschaftlichen Gütekriterien für partizipativ erzeugte Erkenntnisse zu befassen, ist nicht gleichbedeutend damit, die Mechanismen der Qualitätssicherung in der Wissenschaft generell infrage zu stellen oder ersetzen zu wollen, kann aber beinhalten, diese zu verändern. 
wirklicher „Experimente“ innerhalb real vorkommender sozialer Gruppen. Die enge Beziehung zwischen transdisziplinärer Forschung und Aktionsforschung wurde bereits von Gutscher, Hirsch und Werner konstatiert, die unter Rückgriff auf die Aktionsforschung die „Praxisphase“ als konstitutiven Teil transdisziplinärer Forschung behandeln (Gutscher et al. 1996, 69ff.).

Aktionsforschung wiederum wird auch unter dem Begriff „Handlungsforschung“ geführt. Ein wesentliches Merkmal von Handlungsforschung ist die Umsetzung von Ergebnissen in die Praxis und deren Überprüfung anhand dieser Umsetzung: „Die Ergebnisse von Handlungsforschung werden noch während des Forschungsprozesses in die Praxis umgesetzt. Forschung wird als Lern- und Veränderungsprozess sowohl für die Forschenden als auch die erforschten Personen konzipiert. Schlussendliches Ziel ist es, die Kompetenzen der untersuchten Personen so zu erweitern, dass sie ihr gesellschaftliches Interesse selbst vertreten können“ (Schreier 2013, S. 208). Handlungsforschung wird als ein eigenes Forschungsdesign behandelt, das sich von anderen Forschungsdesigns wie Einzelfallanalyse, Dokumentenanalyse, deskriptiver Feldforschung oder Evaluationsforschung unterscheidet (z. B. Seel und Hanke 2015b, S. 802 in Anlehnung insbesondere an Flick et al. 2000b oder Prengel et al. 2008, S. 182). Gemäß Prengel, Heinzel und Carle ist Handlungsforschung darauf ausgerichtet, in der Verbindung von „Forschen und Handeln“ die „Praxis im Einzelfall zu verbessern und darauf, verallgemeinerbare Erkenntnisse für die Übertragung auf ähnlich gelagerte Fälle zu erlangen“" (Prengel et al. 2008, S. 182). Handlungsforschung wird kollaborativ von Personen aus der Wissenschaft und aus der Praxis betrieben, d. h. die Personen aus der Praxis sind ,aktiv am Prozess der Untersuchung beteiligt (...) und [kooperieren] mit Wissenschaftlern" (ebd., S. 182f.). In dieselbe Tradition wie Aktionsforschung und Handlungsforschung gehört das, was im englischen Sprachraum auch ,co-operative inquiry“ genannt wird.

Von Handlungsforschung (und Aktionsforschung) zu unterscheiden ist hingegen das, was Prengel, Heinzel und Carle „Praxisforschung“ nennen. Diese definieren sie als ein in die Praxis „eingelassene[s] Forschen“, das durch die Angehörigen der in dieser Praxis tätigen Berufe „selbst betrieben wird“ (ebd.). Den Unterschied machen sie wesentlich daran fest, dass die Ergebnisse der Forschung im Fall der Praxisforschung nicht darauf abzielen, ,den wissenschaftlichen Erkenntnisstand zu erneuern" (ebd.). Was in der Terminologie von Prengel, Heinzel und Carle „Praxisforschung“ genannt wird, weist eine gewisse Nähe auf zu dem, was andernorts als „Citizen Science“ bezeichnet wird, bei der organisierte oder nichtorganisierte Personen aus der Bevölkerung entweder selbst für sich forschend tätig sind, indem sie einen für sie relevanten Gegenstand untersuchen, aber ohne die Absicht, ihre Befunde in den wissenschaftlichen Diskurs einzuspeisen, oder indem sie Daten liefern für wissenschaftliche Forschungsprojekte, aber ohne dass eine Zusammenarbeit jenseits der Zulieferung stattfindet (s. z. B. Bonn et al. 
2016; Pettibone et al. 2018). Wird Zusammenarbeit aber nicht als etwas Binäres verstanden, sondern als ein Kontinuum mit zunehmender Intensität, lassen sich die Grenzen zwischen „Citizen Science“ bzw. „Praxisforschung“ auf der einen Seite und partizipativer Forschung im Sinne der Handlungsforschung (und Aktionsforschung) auf der anderen Seite nicht immer zwingend scharf ziehen.

Eine große Nähe zu beidem, Aktionsforschung und Handlungsforschung, weist schließlich die Interventionsforschung auf: „Der Ansatz der Interventionsforschung (...) nimmt [zum einen] zur Kenntnis, dass Forschung immer intervenierenden Charakter trägt, selbst wenn in ihrer Ausrichtung zunächst keinerlei direktive Manipulationsabsicht angelegt ist und beobachtet zugleich mögliche (unbeabsichtigte) Einflüsse kritisch und reflexiv. Zum anderen bekennt er sich zu beabsichtigten Interventionen in konkrete Praxisfelder" (Krainer und Lerchster 2012b, S. 10; s. auch Krainer und Lerchster 2012a). Die Beziehung zwischen transdisziplinärer Forschung und Interventionsforschung wiederum wurde in der Literatur auch bereits hergestellt, namentlich durch Krainer und Lerchster: „Interventionsforschung (...) entwickelt Forschungsfragen und teilweise auch Forschungsdesigns häufig in Kooperation mit jenen, die im zu erforschenden System leben und/oder arbeiten und die dementsprechend auch nicht als Forschungsobjekte betrachtet und bezeichnet werden, sondern als Praxis- oder ForschungspartnerInnen. Insofern lässt sich zu Recht auch von ,transdisziplinärer Interventionsforschung' sprechen und gleichwohl Interventionsforschung als eine spezifische Methode transdisziplinärer Forschung darstellen“ (Krainer und Lerchster 2012b, S. 12).

Die generelle Verwandtschaft mit partizipativer Forschung und speziell mit Aktionsforschung (und Handlungsforschung) sowie Interventionsforschung wird in der Literatur nicht nur für transdisziplinäre Forschung konstatiert. Sie spiegelt sich auch in Publikationen zu Reallaborforschung (so etwa in Schneidewind und Singer-Brodowski 2015). Um Hinweise zu erhalten für die Frage nach wissenschaftlichen Gütekriterien für partizipativ erzeugte Erkenntnisse sind also die Diskussionen rund um die Wissenschaftlichkeit der Vorgehensweisen und Ergebnisse in bzw. aus der Aktions- oder Handlungsforschung sowie der Interventionsforschung vielversprechende Quellen.

\section{Die Notwendigkeit, sich systematisch mit Gütekriterien für Methoden zu befassen}

Auf einer sehr grundlegenden Ebene sind partizipative Forschung sowie speziell Aktions-, Handlungs- und Interventionsforschung stark sozialwissenschaftlich geprägt, wobei qualitative Methoden eine prominente Rolle spielen (s. z. B. Prengel et al. 2008, S. 185; von Unger 2014, S. 58). Zusätzlich kommen Methoden 
zum Einsatz, die nicht zum traditionellen Kanon sozialwissenschaftlicher Methoden gehören. Solche Methoden sind insbesondere verschiedene Formen von Moderations- und Kreativverfahren, künstlerische und visuelle Methoden sowie Dialog-Methoden (s. z. B. Defila et al. 2008; Gerhold 2015, S. 111; McDonald et al. 2009; von Unger 2014, S. 10 und S. 57). Letztere wiederum spielen eine herausragende Rolle, weil partizipative Forschung wesentlich darauf angewiesen ist, einen „kommunikativen Raum“ zu schaffen, in dem der Prozess der Wissensproduktion ,über Kommunikation gesteuert wird“ (Bergold und Thomas 2010, S. 335 und S. 337). Dialog-Methoden sind dazu gedacht, dieses Zusammenspiel von kognitiven und sozial-interaktiven Aspekten zu strukturieren. Zudem werden in dieser Forschung - wie in jeder anderen Forschung auch - neue Methoden entwickelt und bestehende angepasst, so dass sich oftmals auch die Grenzen auflösen zwischen ehemals als getrennt erachteten Methoden, wie dies etwa Böhme für die Schulforschung konstatiert, die eine zunehmende Anwendung von „,In-Anlehnung-an'-Methoden oder ,Spielarten' qualitativer Verfahren“" beobachtet bzw. eine ,zunehmende forschungspraktische Aufweichung methodischer Differenzen bei der Datenerhebung, -aufbereitung und -auswertung“ (Böhme 2008, S. 125f.).

Für die Güte sozialwissenschaftlich ausgerichteter und qualitativ vorgehender Forschung ist die intersubjektive Nachvollziehbarkeit entscheidend (und nicht die Reproduzierbarkeit). Das Sicherstellen der Nachvollziehbarkeit dient der Herstellung von Glaubwürdigkeit und prinzipieller Kritisierbarkeit und schafft überhaupt erst die Voraussetzung dafür, dass das produzierte Wissen Eingang finden kann in den wissenschaftlichen Diskurs und dazu beitragen kann, den Erkenntnisstand zu erneuern. Diese generelle Aussage zu qualitativer Forschung gilt auch für partizipative Forschung (z. B. Bergold und Thomas 2010, S. 338) sowie für die Interventionsforschung (Krainer et al. 2012, S. 181). Und schließlich gilt sie auch für inter- und transdisziplinäre Forschung (Defila und Di Giulio 2015). Entsprechend ist Nachvollziehbarkeit auch der Dreh- und Angelpunkt für eine Reflexion der Wissenschaftlichkeit partizipativ erzeugter Erkenntnisse.

Das Herstellen von Nachvollziehbarkeit beinhaltet, dass methodische Entscheidungen dokumentiert und offengelegt werden, so dass diese beurteilt und kritisiert werden können und klar wird, wie welche Daten zustande kommen und an welcher Stelle die Interpretation der Forschenden beginnt (s. z. B. Bogner et al. 2014, S. 93; Flick 2010, S. 398; Mayring 2010; Schüll und Gerhold 2015, S. 94; Steinke 1999, S. 207ff.). Was in welcher Form zu dokumentieren und offenzulegen ist, wird in verschiedenen Disziplinen bzw. disziplinären oder interdisziplinären Wissenschaftsgemeinschaften unterschiedlich gehandhabt. Zudem unterscheidet es sich auch von Methode zu Methode. Unabhängig von diesen Unterschieden lässt sich Folgendes festhalten: Nachvollziehbarkeit entscheidet sich entlang der ausgesprochenen oder unausgesprochenen Regeln darüber, welche Entscheidungen, Grundlagen und Schritte dokumentations- und kommunikationspflichtig sind. 
Nachvollziehbarkeit als Anforderung und Problem verschärft sich nun aber, wenn der traditionelle Kanon sozialwissenschaftlicher Methoden verlassen wird, weil für solche ,andere' Methoden (noch) keine Regeln zur Verfügung stehen, die vorgeben, was es wie zu dokumentieren gilt und wie über das Vorgehen berichtet werden muss. Es erstaunt deshalb nicht, dass, wenn ,ungewöhnliche', neu entwickelte bzw. re-kombinierte und/oder, verflüssigte" Methoden zum Einsatz kommen, „die auch gegenwärtig noch rege geführte Diskussion um Qualitätsstandards bzw. Gütekriterien qualitativer Forschung auf[blüht] (...), in der neben einer Konturierung angewandter Methoden auch die mittlerweile marginalisierte grundlagentheoretisch-methodologische Reflexion des eigenen Forschungsprozesses neu gefordert wird“ (Böhme 2008, S. 125f.). Vor diesem Hintergrund ist es konsequent, wenn von Unger die aus ihrer Sicht derzeit aktuelle Diskussion über Gütekriterien für partizipative Forschung als Versuch einstuft, den Anschluss herzustellen an „wissenschaftliche Qualitätsdiskurse“ (2014, S. 12). Dabei ist zu beachten, dass in der partizipativen Forschung die Güte der Vorgehensweise nicht unreflektiert bzw. allein nach den ,üblichen' Kriterien Objektivität, Validität und Reliabilität beurteilt werden kann. Bezogen auf Handlungsforschung etwa postulieren Prengel, Heinzel und Carle anstelle dieser ,Trias ${ }^{6}$ die „Gütekriterien (...) Praxisrelevanz, Realitätshaltigkeit, Transparenz und Interaktion“ (Prengel et al. 2008, S. 185). Die Bedeutung der Interaktion für die Güte des Vorgehens wird etwa auch von Schreier betont, wenn sie daraus, dass die „Untersuchungsteilnehmerinnen und -teilnehmer (...) als gleichberechtigte Partner der Forschenden angesehen [werden] und (...) an allen Phasen des Forschungsprozesses mit beteiligt [sind]“, schließt, dass „nur solche Methoden [ausgeschlossen sind], die Distanz zwischen den Forschenden und den erforschten Personen schaffen" (Schreier 2013, S. 208f.). Es gilt also, in die Überlegungen darüber, wie und worüber Nachvollziehbarkeit hergestellt werden kann und soll, auch Aspekte einzubauen, die die Interaktion von Forschenden und Praxisakteuren in den Blick nehmen.

Gleichzeitig lässt sich leider beobachten, dass die Praxis transdisziplinärer (aber auch interdisziplinärer) Forschung oftmals Defizite aufweist hinsichtlich der Nachvollziehbarkeit ihrer Ergebnisse, und zwar insbesondere ihrer Syntheseergebnisse, weil das methodische Zustandekommen solcher Ergebnisse häufig gar nicht oder lediglich oberflächlich beschrieben wird (s. Defila und Di Giulio 2015). Ein wesentlicher Grund für dieses Phänomen dürfte das Fehlen methodischer Gütekriterien sein bzw. die derzeit noch ausstehende Diskussion darüber, wie Nachvollziehbarkeit, und damit Glaubwürdigkeit und prinzipielle Kritisierbarkeit, hergestellt werden kann und soll. Dies ist - nebst den Zweifeln an der Gültigkeit partizipativ erzeugten Wissens innerhalb der Wissenschaft (s. Kapitel 1) -, ein zweiter guter Grund, sich mit der Frage der wissenschaftlichen Gütekriterien für partizipativ erzeugte Erkenntnisse transdisziplinärer Forschung zu befassen - und dabei auf Methoden zu fokussieren. 
Ein dritter Grund, der dafür spricht, sich mit wissenschaftlichen Gütekriterien rund um Methoden der partizipativen Wissenserzeugung in transdisziplinärer Forschung zu beschäftigen, ist der im Feld transdisziplinärer (aber auch interdisziplinärer) Forschung und neuerdings auch im Feld der Reallaborforschung bestehende forschungspraktische Bedarf. In Kontexten, in denen ein Methodenmix sinnvoll ist und in denen dabei nicht allein die traditionellen sozialwissenschaftlichen Methoden zum Zuge kommen bzw. in denen solche Methoden auch neu re-kombiniert werden, müssen Forschende für eine angemessene Methodenwahl (und -anpassung) auf einen „umfangreichen Pool an verfügbaren Methoden“ zurückgreifen können (Gerhold 2015, S. 111). Das wiederum setzt voraus, dass Methoden so aufbereitet und zur Verfügung gestellt werden, dass sie nach wissenschaftlichen Gesichtspunkten ausgewählt und umgesetzt werden können und klar ist, wie deren Wahl und Umsetzung sowie die damit erzeugten Ergebnisse dokumentiert und kommuniziert werden sollen, damit Wahl und Umsetzung der Methoden sowie die erzeugten Ergebnisse einer wissenschaftlichen Qualitätsprüfung unterzogen werden und so zu Wissen führen können, das als intersubjektiv gesichert erachtet werden kann. Der forschungspraktische Bedarf lässt sich also ohne Auseinandersetzung mit wissenschaftlichen Gütekriterien für Methoden nicht decken.

Der forschungspraktische Bedarf nach einem Methoden-Pool spiegelt sich in einer zunehmenden Zahl handbuchartiger Werke, in denen im weitesten Sinne Methoden und Techniken für die Gestaltung (inter- und) transdisziplinärer Prozesse zur Verfügung gestellt werden (s. z. B. Bergmann et al. 2010; Defila et al. 2006; Loibl 2005; Niederberger und Wassermann 2015; Nolting et al. 2008; Pohl und Hirsch Hadorn 2006; Rabelt et al. 2007; Schophaus et al. 2004; von Blanckenburg et al. 2005; Online-Methodensammlungen). Aus der Perspektive, die in diesem Beitrag eingenommen wird, weisen solche Werke jedoch zwei Defizite auf: Erstens unterscheiden sie noch zu wenig pointiert zwischen Methoden der Wissenserzeugung (was bei inter- und transdisziplinärer Forschung wesentlich die Synthesebildung beinhaltet, d. h. das Erzeugen neuen Wissens durch Integration) und anderen einer methodischen Herangehensweise zugänglichen Bereichen der (inter- und) transdisziplinären Arbeit wie z. B. der Gestaltung der Teamdynamik oder dem Aufbau von Vertrauen und gegenseitigem Verständnis. Bereiche wie diese methodisch gut zu gestalten, ist sicher Voraussetzung für das Erzeugen integrierten Wissens, aber dennoch sind die dabei verwendeten Methoden nicht als Methoden der Wissenserzeugung zu adressieren, es sei denn, sie seien Teil einer entsprechenden Methoden-Kombination. Zweitens beruht die Art und Weise, wie die Methoden in solchen Werken vorgestellt werden, nicht auf einer reflektierten (und transparenten) Liste von wissenschaftlichen Gütekriterien für Methoden der Wissenserzeugung. Das Erste ist aber auch nicht das Ziel solcher Werke, und das Zweite entsprechend nicht deren Fokus. In diesen beiden Punkten einen Schritt weiterzukommen, erscheint aber nötig als Beitrag zur Wissenschaft- 
lichkeit der in transdisziplinärer Forschung bzw. in Reallaborforschung erzeugten Ergebnisse und damit als Beitrag zur Verbesserung der Voraussetzungen dafür, dass diese Ergebnisse vermehrt Eingang finden in den jeweiligen thematischen wissenschaftlichen Diskurs.

\section{Ein Vorschlag für wissenschaftliche Gütekriterien für Methoden der partizipativen Wissenserzeugung in der transdisziplinären Forschung}

In diesem Kapitel wird ein Vorschlag unterbreitet für Gütekriterien, die in der transdisziplinären Forschung außerhalb wie innerhalb von Reallaboren berücksichtigt werden sollten

- bei der Aufbereitung, d. h. beim Zur-Verfügung-Stellen, von Methoden der partizipativen Wissenserzeugung: Was muss mit Blick auf eine gut begründete Methodenwahl sowie die korrekte Umsetzung und Dokumentation einer Methode erklärt werden, wenn eine Methode zuhanden Dritter aufbereitet wird?

- bei der Wahl (inkl. Entwicklung und Kombination) solcher Methoden: Was muss bei der Wahl von Methoden bzw. bei der Anpassung und Entwicklung von Methoden berücksichtigt werden?

- bei der Umsetzung solcher Methoden: Was muss bei der Umsetzung einer Methode befolgt werden, damit die Umsetzung korrekt ist und zu einem belastbaren Ergebnis führt?

- bei der Dokumentation und Reflexion des methodischen Vorgehens: Was muss während der Methodenwahl und während der Umsetzung einer Methode dokumentiert werden, damit Vorgehen und Ergebnis nachvollziehbar sind, worüber sollte während der Durchführung reflektiert werden, und was sollte im Vorfeld (z. B. in Anträgen) oder im Nachgang (z. B. in Publikationen) expliziert werden?

Der Vorschlag ist entstanden aus der Auswertung von Publikationen, die sich dazu äußern, welche Gesichtspunkte bei der Methodenwahl entscheidend sind, und die zugleich wissenschaftlich fundierte, aber auf die praktische Anwendung ausgerichtete Beschreibungen von Methoden enthalten. Dies sind traditionellerweise Methoden-Handbücher. Bei der Literaturanalyse fand, mit wenigen Ausnahmen, eine Beschränkung statt auf sozialwissenschaftliche Methoden-Handbücher, wobei solche bevorzugt wurden, die sich nicht nur zu einer einzigen Methode äußern, sondern einen Überblick über verschiedene Methoden geben (namentlich Baur und Blasius 2014a; Flick et al. 2000a; Gerhold et al. 2015; Helsper und 
Böhme 2008; Hussy et al. 2013; Mayring 2010; Mey und Mruck 2010; Seel und Hanke 2015a). In diesen wiederum wurden ausschließlich die Ausführungen zu qualitativen Methoden berücksichtigt. Berücksichtigt wurden zudem Ausführungen $\mathrm{zu}$ partizipativer Forschung und $\mathrm{zu}$ Interventionsforschung. Nachstehend wird zuerst auf die Literaturanalyse eingegangen (Abschnitt 4.1), anschließend wird der Kriterien-Vorschlag vorgestellt (Abschnitt 4.2).

\subsection{Gütekriterien für Methoden der partizipativen Wissenserzeugung in der Literatur}

Wie alle anderen Methoden der Wissenserzeugung in der Forschung auch lassen sich Methoden der partizipativen Wissenserzeugung definieren als ein planmäßiges Verfahren zur Gewinnung wissenschaftlicher Erkenntnisse. Eine Methode ist ein beschreibbarer Weg zur Beantwortung einer bestimmten Frage. Dieser Weg besteht aus einer endlichen Folge von Handlungen, deren Abfolge vorgegeben ist. Je nach Methode sind die Vorgaben für diese Abfolge mehr oder weniger fix und die Vorgaben für die Handlungen mehr oder weniger detailliert. Aus dieser generellen Umschreibung dessen, was Methoden der Wissenserzeugung sind, ergibt sich zwangsläufig, dass bei der Aufbereitung einer Methode sowohl auf die Handlungen einzugehen ist, die in der Umsetzung der Methode zu vollziehen sind (inkl. der Reihenfolge, in der diese aufeinander folgen sollen), als auch auf das Ziel, das mit der Methode erreicht werden kann. Letzteres ist insbesondere für die Wahl von Methoden entscheidend (z. B. Cuhls 2015, S. 86; Prengel et al. 2008, S. 182), und zwar auch in der partizipativen Forschung (z. B. Bergold und Thomas 2010, S. 338; von Unger 2014, S. 56). Die Bedeutung der Ziele mit Blick auf die Methodenwahl wird in der Literatur zumeist stillschweigend vorausgesetzt, und manchmal werden dafür auch andere Bezeichnungen gewählt. So streichen einige Autorinnen und Autoren die Bedeutung der Frage hervor, die mit Hilfe einer Methode beantwortet werden soll (z. B. Baur und Blasius 2014b; Gerhold 2015; Lerchster 2012; Przyborski und Wohlrab-Sahr 2014), oder nennen die spezifischen Ergebnisse, die mit einer Methode erzeugt werden können (z. B. Gerhold 2015). Wieder andere schreiben, die Methodenwahl hänge von der „Forschungslage" ab, also davon, was bereits bekannt sei und was es noch herauszufinden gelte (z. B. Gutscher et al. 1996). Ebenso zwangsläufig ergibt sich daraus, dass beides, das verfolgte Ziel (bzw. die zu beantwortende Frage) und die vorgenommenen Handlungen, Teil auch der Dokumentation von Vorgehen und Ergebnis sein muss.

Jede Methode kann nur bestimmte Arten von Antworten liefern und schließt damit andere Arten von Antworten aus. Methoden unterscheiden sich also auch dahingehend, welcher (vorwissenschaftliche) Gegenstand sich damit erfassen lässt und welche Art Daten über diesen erzeugt werden können. Es ist selbstre- 
dend, dass bspw. standardisierte Fragebögen, biographische Interviews, DelphiVerfahren oder Interventionen je eine andere Art von Daten, und damit von Ergebnissen, erzeugen. Dasselbe gilt aber auch, wenn in transdisziplinären Designs Methoden eingesetzt werden, mit denen z. B. die Grundannahmen der Beteiligten aufeinander bezogen werden, mit denen an einem gemeinsamen Verständnis zentraler Begriffe gearbeitet wird oder mit denen eine mehrperspektivische Beschreibung des zu untersuchenden Problems entwickelt wird. Keine Methode kann das ,Ganze“ eines vorwissenschaftlichen Gegenstands (Phänomens, Problems) in den Blick nehmen, d. h. jede Methode fokussiert zwangsläufig auf bestimmte Aspekte (Eigenschaften) vorwissenschaftlicher Gegenstände und blendet andere aus. Der so in seiner realweltlichen Komplexität reduzierte vorwissenschaftliche Gegenstand wiederum bildet den wissenschaftlichen Gegenstand, über den Daten erzeugt werden können (und gleichzeitig bestimmt die vorgenommene Reduktion, welche Art Daten erzeugt werden können). Aus der Perspektive der Methodenwahl müssen Methoden nicht nur zum angestrebten Ziel, sondern auch zum Gegenstand, der untersucht werden soll, passen, d. h. zum Phänomen (oder ,Datenträger'), das erfasst werden soll (z. B. Baur und Blasius 2014b; Breuer 2010; Gerhold 2015; Przyborski und Wohlrab-Sahr 2014). Um sich ein angemessenes Bild der diesbezüglichen Leistung einer Methode verschaffen zu können, muss klar sein, welcher Gegenstand damit erfasst werden kann, d. h. welche Art Daten (und damit Ergebnisse) mit der Methode erzeugt werden können. Um zum einen eine fundierte Methodenwahl zu erlauben und um zum anderen die durch die gewählte(n) Methode(n) vorgenommene Reduktion des vorwissenschaftlichen Gegenstands einer Beurteilung zugänglich zu machen, gehören damit Ausführungen zum Gegenstand sowohl zur Aufbereitung einer Methode als auch zur Dokumentation von Vorgehen und Ergebnis. Das beinhaltet die Angabe des vorwissenschaftlichen Gegenstands sowie die Angabe der Aspekte dieses Gegenstands, auf die eine Methode fokussiert.

Ein letztes Kriterium, das generell für Methoden der Wissenserzeugung in der Forschung, und damit auch für Methoden der partizipativen Wissenserzeugung in transdisziplinärer Forschung, gilt, betrifft die Theorie: Methoden lassen sich nicht auf den ,technischen' Aspekt der Datenerzeugung reduzieren. Sie sind immer mindestens implizit auch theorie- und wertgeladen (s. dazu z. B. Potthast 2015), d. h. sie setzen bestimmte Theorien (und Wertsysteme) voraus bzw. sind mit bestimmten Theorien leichter vereinbar und mit anderen schwerer oder auch gar nicht. Die mit einer Methode verknüpften Theorien wiederum bestimmen auch den wissenschaftlichen Gegenstand, der mit der Methode erfasst werden kann, da der wissenschaftliche Gegenstand definiert werden kann als die Summe der theorierelevanten Eigenschaften (Aspekte) eines vorwissenschaftlichen Gegenstands. Die zentrale Bedeutung von Theorien betont z. B. auch Böhme, wenn sie dafür argumentiert, das methodologische Problembewusstsein dafür zu schärfen, „,dass etablierten Methoden teilweise gänzlich differente Wirklichkeitsvorstellungen 
immanent sind, von denen die Forschungspraxis nicht abstrahieren kann" (2008, S. 126). Daraus ergibt sich für die Methodenwahl, dass die Methoden auch in theoretischer Hinsicht passen müssen. Das wiederum hat zwei Ausprägungen: Die mit einer Methode verknüpften Theorien müssen erstens zu den Theorien passen, die den Hintergrund der zu beantwortenden Forschungsfrage bilden. Zweitens müssen sie zu den Theorien passen, die von den am Forschungsvorhaben beteiligten Personen bevorzugt verwendet werden. Darüber, dass (erkenntnistheoretische) Vorannahmen über, die Welt' und das Menschenbild sowie präferierte Theorien bei der Methodenwahl eine entscheidende Rolle spielen, besteht eine große Einigkeit (z. B. Altrichter und Feindt 2008, S. 449; Baur und Blasius 2014b; Bergold und Thomas 2010; Breuer 2010; Bogner et al. 2014, S. 93; Scheele und Groeben 2010, S. 506; Lerchster 2012; Przyborski und WohlrabSahr 2014; von Unger 2014, S. 56). Die Angabe, welche Theorien mit der Methode untrennbar verknüpft sind, muss daher zwingend Teil der Aufbereitung einer Methode sein - im Idealfall auch eine Angabe dazu, welche (Art) Theorien sich damit schwer oder gar nicht verbinden lassen bzw. welche sich damit leicht verbinden lassen. Ausführungen zum theoretischen Hintergrund der verwendeten Methode(n) sollten aber auch Teil der Dokumentation von Vorgehen und Ergebnis sein.

Werden Einzelmethoden kombiniert bzw. zu neuen Methoden re-kombiniert, ist auf die genannten Kriterien Handlungsabfolge, Ziel, Gegenstand und Theorie für die einzelnen Methoden wie auch für die Kombination der Methoden einzugehen. Damit wird gewährleistet, dass die Stimmigkeit des ,Pakets‘ sowohl bei der Methodenwahl als auch bei der Beurteilung von Vorgehen und Ergebnis berücksichtigt werden kann (das Kriterium der Stimmigkeit bei der Kombination von Methoden streicht z. B. von Unger (2014, S. 58) hervor).

Ethische Aspekte, d. h. die Frage, welche Handlungen zulässig sind bzw. welche Vorkehrungen für ein ethisch korrektes Handeln zu treffen sind, spielen bei der Wahl und Umsetzung von Methoden immer eine Rolle, insbesondere bei solchen, in denen mit Menschen gearbeitet wird. Entsprechende Angaben bei der Aufbereitung von Methoden und bei der Dokumentation von Vorgehen und Ergebnis sind ein Teil dessen, worauf beim Kriterium Handlungsabfolge einzugehen ist. Bei partizipativer Forschung sind zusätzliche ethische Aspekte zu beachten (z. B. Baur und Blasius 2014b; Altrichter und Feindt 2008, S. 460) bzw. müssen anerkannte Regeln wie etwa die, dass im Vorfeld ein informiertes Einverständnis seitens von Personen, die einer Methode ,ausgesetzt werden', vorliegen muss, an die Besonderheiten partizipativer Forschungsprozesse (z. B. nur bedingte Planbarkeit) angepasst werden (z. B. von Unger 2014, S. 91ff). Ähnliches gilt für das Gebot der Transparenz, d. h. für die Frage, wer zu welchem Zeitpunkt über was informiert werden sollte (s. dazu aber auch Quint et al. 2018). Eine besondere Bedeutung kommt Aspekten rund um die Beziehung und Interaktion der Beteilig- 
ten zu. Auf die soziale Konstellation und die Interaktion in einem partizipativen Vorgehen gehen verschiedene Autorinnen und Autoren ein, und diese betonen unterschiedliche, sich ergänzende Punkte - und diese Punkte wiederum wirken sich nicht nur auf die Handlungen aus, die bei der Umsetzung einer Methode auszuführen sind, sondern auch auf die Kriterien Ziel, Gegenstand und Theorie (und werden umgekehrt von diesen Kriterien beeinflusst).

Drei der Facetten, die in der Literatur betont werden, betreffen die Beziehung und Interaktion zwischen Forschenden und Praxisakteuren:

- Die erste Facette ist die Notwendigkeit, eine gewisse Distanz herzustellen bzw. zu erhalten sowohl zwischen den Forschenden und den Praxisakteuren, mit denen zusammengearbeitet wird, als auch zwischen den Forschenden und dem Praxisfeld, in dem forschend gehandelt wird. Die Beziehung zwischen den Forschenden und den Praxisakteuren bzw. zwischen den Forschenden und dem Praxisfeld ist laufend zu reflektieren (z. B. Bergold und Thomas 2010, S. 238; Prengel et al. 2008, S. 186f.). Nach Altrichter und Feindt geht es bei partizipativer Forschung um ,eine immer wieder herzustellende Balance zwischen Distanz und Involvierung und wiederum distanzierter Reflexion dieser Involvierung“" (2008, S. 459).

- Die zweite Facette ist die Notwendigkeit, sich damit auseinanderzusetzen, welche Dynamik die Forschungssituation im Praxisfeld erzeugt, d. h. wie das Forschungsvorhaben bzw. das Handeln der daran beteiligten Personen das Handeln und die Interaktionen im Praxisfeld beeinflussen (z. B. Breuer 2010; Lerchster 2012, S. 49ff.; Prengel et al. 2008, S. 186). Gleichzeitig gilt es, sensibilisiert zu sein dafür und zu verhindern, dass Methoden der Wissenserzeugung, politisch` instrumentalisiert werden (können).

- Zur Gestaltung des Verhältnisses zwischen Forschenden und Praxisakteuren gehört bei partizipativer Forschung als dritte Facette, dass nicht der Fehler gemacht wird, Praxisakteure zu Forschenden machen zu wollen oder umgekehrt. ${ }^{5}$ Dies betonen etwa Prengel, Heinzel und Carle, die sagen, es sei ,also gar nicht ,Sinn der Sache“, dass Praktiker zu Wissenschaftlern werden oder

5 Damit ist nicht gemeint, Praxisakteure sollten sich nicht forschend betätigen. In der transdisziplinären Forschung geht es ja gerade darum, dass sich Praxisakteure substantiell an der Forschung beteiligen (s. Kapitel 1). Sie beteiligen sich daran aber als ,noncertified experts“ und nicht als „,ertified experts“, d. h. indem sie sich an der Tätigkeit ,Forschen' beteiligen, werden sie nicht zu ,Forschenden' in der Bedeutung, wie sie hier zugrunde gelegt wird, d. h. sie werden nicht zu Forschenden des Wissenschaftssystems. Diese Unterscheidung zwischen Tätigkeit und Personen mag haarspalterisch scheinen und sich sperrig anfühlen, sie ist aber hilfreich, um nicht zu verwischen, dass die gemeinsame Tätigkeit auf der Zusammenführung der unterschiedlichen und sich ergänzenden Expertisen beruht (s. dazu auch Di Giulio et al. 2016, S. 272ff.). 
umgekehrt“, und Wert legen darauf, dass „,die Erkenntnismöglichkeiten“ vielmehr „gerade aus dem Prozess der Reflexion der jeweils anderen Perspektive [erwachsen]“ (2008, S. 187).

Daraus lässt sich folgern, dass Ausführungen dazu erforderlich sind, welche unterschiedlichen Rollen und welche Beziehung zwischen Forschenden und Praxisakteuren eine Methode voraussetzt bzw. wie sich die Beziehung zwischen Forschenden und Praxisakteuren in der Umsetzung einer Methode gestaltet bzw. welche diesbezüglichen Herausforderungen sich stellen, und zwar sowohl bei der Aufbereitung von Methoden als auch bei der Dokumentation von Vorgehen und Ergebnis.

Drei der Facetten, die in der Literatur betont werden, betreffen die Beziehung und Interaktion der Praxisakteure untereinander:

- Die erste dieser Facetten betrifft den Charakter der Kommunikationssituation, der in einem partizipativen Vorgehen geschaffen wird. Es ist unabdingbar, Beteiligung und offene Äußerungen sicherzustellen und soziale Ausschlussformen zu vermeiden (z. B. Bergold und Thomas 2010, S. 238; Prengel et al. 2008, S. 186f.). Dies betonen auch Scheele und Groeben unter Rückbezug auf die „ideale Sprechsituation“ nach Habermas, wenn sie schreiben, es seien „Bedingungen der Möglichkeit von Wahrhaftigkeit zu schaffen; Bedingungen, aufgrund derer die Person unverzerrt über ihre Innensicht $\mathrm{zu}$ sprechen vermag" (2010, S. 508).

- Verwandt, aber nicht identisch ist die zweite Facette, die Machtstrukturen (innerhalb und außerhalb des Forschungsvorhabens) adressiert und wonach zu fragen ist, ,in welcher Weise die Machtstruktur das Teilnehmen von Menschen an Entscheidungen und Aktionen erlaubt, verhindert oder unterdrückt, und welche Position die Akteure in dieser Machtstruktur einnehmen, über welche Machtressourcen sie verfügen oder welche ihnen ermangeln. Für die partizipative Forschung wird dies wichtig, weil Macht oft nicht direkt sichtbar wird; vor allem strukturelle Macht setzt sich hinter dem Rücken der Beteiligten durch" (Bergold und Thomas 2010, S. 336).

- Die dritte Facette schließlich betrifft die Beziehung zwischen den Praxisakteuren und dem Praxisfeld, in dem forschend gehandelt wird. Die von Altrichter und Feindt hervorgestrichene Notwendigkeit, die „Balance zwischen Distanz und Involvierung und wiederum distanzierter Reflexion dieser Involvierung“ immer wieder herzustellen, gilt nicht nur bezogen auf die Forschenden, sondern auch bezogen auf die Praxisakteure (2008, S. 459).

Daraus lässt sich folgern, dass Ausführungen dazu nötig sind, wie sich eine Methode auf die Praxisakteure auswirkt, welche Beziehung zwischen den Praxisakteuren eine Methode voraussetzt bzw. wie sich die Beziehung zwischen den 
Praxisakteuren in der Umsetzung einer Methode gestaltet bzw. welche diesbezüglichen Herausforderungen sich stellen, und zwar sowohl bei der Aufbereitung von Methoden als auch bei der Dokumentation von Vorgehen und Ergebnis.

Die Voraussetzungen der Partizipation, in der Welt ' sind ein letztes Kriterium, das spezifisch ist für Methoden der partizipativen Wissenserzeugung. Natürlich gilt generell, dass bei der Wahl und Umsetzung von Methoden auch entscheidend ist, welche Rahmenbedingungen und Ressourcen für die Forschung bestehen (innerhalb des Projekts wie im Feld), weil der Einsatz einer Methode immer auch von spezifischen Voraussetzungen und Anforderungen abhängt, die dafür gegeben sein müssen (z. B. Gerhold 2015). Spezifisch für partizipative Forschung ist, dass dabei auch Rahmenbedingungen und Ressourcen zu berücksichtigen sind, die die Voraussetzungen der Partizipation in den Blick nehmen. Dazu gehören zum einen hemmende/fördernde strukturelle, institutionelle oder organisationale Rahmenbedingungen im Praxisfeld bzw. bei den Praxisakteuren, die bei der Wahl oder der Umsetzung einer Methode zu beachten sind. Zum anderen gehören dazu Aspekte wie die für die Umsetzung einer Methode erforderlichen Ressourcen und Kompetenzen seitens von Praxisakteuren sowie der Zugang von Praxisakteuren zu einer Methode, also z. B. die Verständlichkeit einer Methode für Praxisakteure oder die Frage, inwieweit eine Form der Datenerhebung den Ausdrucksmöglichkeiten von Praxisakteuren entspricht (z. B. Bergold und Thomas 2010, S. 336ff.; von Unger 2014, S. 56 und S. 86ff). Die Passung zwischen einer Methode und den Kompetenzen der Praxisakteure ist auch von Bedeutung, um der Falle zu entgehen, Praxisakteure zu Forschenden machen zu wollen (s. auch Fn. 5), und die etwa von Prengel, Heinzel und Carle so beschrieben wird: „Der Umgang mit vorhandenen Ausgangs-, Qualifikations- und Funktionsunterschieden im Kooperationsprozess erwies sich allerdings (...) als kompliziert. Der Anspruch der gleichberechtigten Kooperation scheint dazu zu verleiten, bei Forschern und Praktikern die gleichen Kompetenzen vorauszusetzen oder den Erwerb derselben zum Ziel zu erheben" (2008, S. 186; s. dazu auch den ähnlichen Befund von Di Giulio et al. 2016, S. 272ff.). Daraus ergibt sich, dass die Auseinandersetzung mit den Voraussetzungen der Partizipation ein weiteres Element darstellt, auf das bei der Aufbereitung einer Methode sowie bei der Dokumentation von Vorgehen und Ergebnis einzugehen ist (s. dazu auch Eckart et al. 2018).

\subsection{Katalog von Gütekriterien für Methoden der partizipativen Wissenserzeugung in der transdisziplinären Forschung}

Der nachstehende Vorschlag für Gütekriterien für Methoden der partizipativen Wissenserzeugung in der transdisziplinären Forschung spiegelt die Ergebnisse der Literaturanalyse (s. Abschnitt 4.1). 
Drei Kriterien nehmen Ziel und Kontext von Methoden in den Blick, acht Kriterien die Umsetzung von Methoden im engeren Sinne und zwei Kriterien übergeordnete Erfahrungen im Einsatz von Methoden. Die einzelnen Kriterien sind als Fragen formuliert (und kursiv gesetzt). Zu jedem Kriterium erfolgt eine Erläuterung des Kriteriums, indem angegeben wird, welche Aspekte das Kriterium abdeckt. Die Perspektive, die bei der Erläuterung eingenommen wird, ist die der Aufbereitung von Methoden (s. dazu die einleitenden Ausführungen zu Kapitel 4$){ }^{6}$

\section{Der Blick auf Ziel und Kontext}

\section{Zeitpunkt im Forschungsdesign/Projektablauf - wann?}

- Ausführungen dazu, wann in einem Projektverlauf sich die Methode besonders gut einsetzen lässt. Das ist nicht absolut zu verstehen, sondern relativ zu den Arbeiten und zum Prozess in einem Projekt (z. B. wenn die Ziele und Fragen bereits definiert bzw. noch nicht definiert sind; wenn der Zeitpunkt gekommen ist, sich der Wissensbestände im Projekt zu vergewissern; wenn eine geteilte Problemsicht erarbeitet werden muss; wenn in Teilprojekten genügend Wissen erarbeitet wurde, das es zusammenzuführen lohnt; wenn im Projekt genügend Vertrauen zwischen den Beteiligten aufgebaut ist, dass damit gerechnet werden darf, dass ein offener Austausch stattfindet).

- Falls möglich: Ausführungen dazu, wann in einem Projektverlauf sich die Methode gar nicht einsetzen lässt.

Angestrebtes Ziel - wozu?

- Ausführungen dazu, welchem Ziel die Methode dient bzw. welche Frage damit beantwortet werden kann bzw. welche Art Ergebnis damit erzeugt werden kann, welche Art Wissen damit produziert werden kann (z. B. Bezug nehmend auf Systemwissen, Zielwissen, Transformationswissen $\left.{ }^{7}\right)$.

- Bei einer Methode für die Konsensbildung ${ }^{8}$ oder Synthesebildung: Ausführungen dazu, welche Art integriertes Wissen damit erzeugt werden kann (z. B.

6 Aus den Ausführungen in Abschnitt 4.1 wird deutlich, dass diese Kriterien eng zusammenhängen. Der Vorschlag besteht deshalb nicht darin, dass bei der Aufbereitung von Methoden auf jedes einzelne der Kriterien getrennt eingegangen wird, sondern lediglich darin, dass die entsprechenden Informationen gegeben werden.

7 Zu diesen Wissensarten s. z. B. CASS und ProClim- (1997).

8 Zum Begriff der Konsensbildung in einem inter- oder transdisziplinären Forschungsprojekt s. Defila, Di Giulio und Scheuermann (2006). 
eine mehrperspektivische Analyse eines Phänomens; eine Meta-Theorie; ein integriertes Begriffssystem; eine gesamtheitliche Systemdarstellung).

\section{Partizipationsgrundlage - wie sieht das Feld aus?}

- Ausführungen dazu, welche Voraussetzungen der Partizipation im Praxisfeld bzw. bei den an der Forschung partizipierenden Praxisakteuren bestehen sollten, damit die Methode gut und zielführend umgesetzt werden kann, und welche Rahmenbedingungen im Praxisfeld besonders förderlich bzw. hemmend sind. Ausführungen dazu, welche Ressourcen, Kompetenzen und Bereitschaften seitens der Praxisakteure für die Umsetzung der Methode nötig sind, und wie zugänglich und verständlich die Methode für Praxisakteure ist, d. h. welche Art Akteure mit der Methode erreicht werden kann, wer davon abgeschreckt werden könnte.

- Hinweise darauf, wie ggf. mit Qualifikations- und Funktionsunterschieden oder mit Konflikten im Praxisfeld umgegangen werden kann.

- Bei Bedarf: Ausführungen dazu, welche auf die Methode bezogene Unterstützung/Schulung für Praxisakteure angezeigt sein könnte.

\section{Der Blick auf die Umsetzung}

\section{Ablauf und Tätigkeiten - wie?}

- Ausführungen dazu, welche Handlungen in der Umsetzung der Methode in welcher Reihenfolge stattfinden müssen, und welche Voraussetzungen gegeben sein bzw. geschaffen werden müssen, um die einzelnen Handlungen auszuführen (inkl. ggf. besondere Techniken, Infrastrukturen, Software, Räumlichkeiten, Material etc.).

- Erläuterung des korrekten Vorgehens bei der Durchführung der einzelnen Schritte und Hinweise auf besondere Gefahren und Stolperfallen, die möglicherweise übersehen werden.

- Erläuterung dazu, wie Daten aus Zwischenschritten zu verarbeiten sind, und ggf. auch dazu, wie sie weiterverwendet werden können.

- Falls sinnvoll: Darlegung von Varianten des Vorgehens und nach Möglichkeit Hinweise darauf, was bei der Entscheidung berücksichtigt werden könnte bzw. sollte. 
Ethik - was darf man?

- Erläuterung der aus ethischer Sicht relevanten Aspekte in der Umsetzung der Methode sowie in der Aufbereitung und Weiterverwendung der gewonnenen Daten/Ergebnisse. Erläuterung der aus ethischer Sicht zu treffenden Vorkehrungen zum Schutz der direkt oder indirekt an der Methodenumsetzung beteiligten bzw. von der Methodenumsetzung betroffenen Personen.

- Ausführungen dazu, welche (Art) Handlungen aus ethischen Gründen geboten sind, welche ethisch bedenklich sind, und welche ethisch nicht zulässig sind. Falls vorhanden: Erklärung der bei welchem Schritt zu beachtenden ethischen Prinzipien, Regeln und Kriterien.

- Ausführungen dazu, wo und wann in der Umsetzung der Methode möglicherweise Grenzen zu ziehen sind, um die Praxisakteure keinen Sachzwängen zu unterwerfen, die sie im Vorfeld nicht abschätzen konnten.

(Vorwissenschaftlicher) Gegenstand - was wird erfasst?

- Ausführungen dazu, welcher vorwissenschaftliche Gegenstand (bzw. welche Art Gegenstände) mit der Methode in den Blick genommen werden kann (bzw. können) bzw. Erläuterung der Kriterien, anhand derer geprüft werden kann, ob die Methode für den zu untersuchenden Gegenstand infrage kommt.

- Ausführungen dazu, welche Aspekte (Eigenschaften) eines vorwissenschaftlichen Gegenstands sich mit der Methode erfassen lassen, d. h. welche Art Daten erzeugt werden können. Erklärung, ob sich daraus spezielle und möglicherweise nicht evidente Konsequenzen ergeben für die Verarbeitung und Weiterverwendung der Daten.

- Falls sinnvoll und möglich: Erläuterung, auf welche Art vorwissenschaftliche Gegenstände die Methode auf keinen Fall angewendet werden sollte bzw. zur Erfassung welcher Aspekte von Gegenständen die Methode sicher nicht geeignet ist.

Zugrunde liegende Vorstellungen - welches Weltbild, welches Menschenbild, welche Theorie?

- Ausführungen dazu, welche (Arten von) theoretischen Positionen mit der Methode zwingend verknüpft sind, und, falls möglich, Ausführungen dazu, welche Vorbehalte diesen Theorien gegenüber möglicherweise bestehen können. Dabei den Blick sowohl auf die Gegenstände richten (z. B. „die Methode geht von der Theorie aus, dass Narrative wirkmächtig sind“ oder „die Methode baut auf der Kapitalstock-Theorie auf") als auch auf die in die Umsetzung der Methode einbezogenen Menschen (z. B. „die Methode geht davon aus, dass 
Menschen komplexe Entscheidungen treffen können“ oder „die Methode geht davon aus, dass Menschen willens sind, auch mit Personen, die gegenteilige Positionen vertreten, einen sachlichen Diskurs zu führen").

- Erläuterung, welchem theoretischen Diskurs die Methode entstammt (welches ,Weltbild“ ihren Hintergrund bildet).

- Falls möglich: Ausführungen dazu, welche (Arten von) Theorien mit der Methode besonders leicht verbunden werden können, und dazu, welche mit der Methode nicht kompatibel sind, obwohl man diesen Eindruck haben könnte.

\section{Kombination - wie sind die Methoden kombiniert?}

- Falls es sich um eine Methode handelt, die aus der Re-Kombination bestehender Methoden hervorgegangen ist, bzw. um eine Kombination von Methoden: Ausführungen dazu, aus welchen Einzel-Methoden bzw. Teilen von Methoden die Kombination besteht und aus welchen Gründen diese kombiniert wurden. Erläuterung dazu, was die Konsistenz der Methoden-Kombination ausmacht (dabei auf Ziel, Gegenstand, Theorien und soziale Interaktion eingehen). Ausführungen dazu, worauf bei der Umsetzung zu achten ist, um nicht disparate Teilergebnisse zu erzeugen, die nicht zueinander passen bzw. nicht weiterverwendet werden können.

- Falls es sich um eine Einzelmethode handelt: Erläuterung, ob es (Arten von) Methoden gibt, die mit der Methode besonders gut kombinierbar sind (welche also besonders gut anschließen bzw. sich besonders eignen im Vorfeld). Falls möglich: Erläuterung, ob es (Arten von) Methoden gibt, die mit der Methode gar nicht kombinierbar sind, bei denen jemand aber leicht auf die Idee kommen könnte, sie damit zu kombinieren.

\section{Beziehung zwischen Forschenden und Praxisakteuren - wie Nähe und Distanz gestalten?}

- Ausführungen dazu, wem bei der Umsetzung der Methode welche Rolle zukommt, d. h. welche Entscheidungen weshalb von Forschenden und welche weshalb von Praxisakteuren getroffen werden sollten, welche Handlungen von Forschenden und welche von Praxisakteuren ausgeführt werden sollten.

- Ausführungen dazu, welche Beziehung zwischen Forschenden und Praxisakteuren die Methode voraussetzt und wie sie diese Beziehung verändert. Hinweise darauf, wo bei der Umsetzung der Methode die Gefahr aufkommen könnte, dass die nötige Distanz zwischen Forschenden und Praxisakteuren bzw. zwischen Forschenden und Praxisfeld verloren gehen könnte, und wie sich diese Gefahr verringern lässt. Bei Bedarf: Ausführungen dazu, wo in der 
Umsetzung der Methode besonders darauf zu achten ist, die Unterschiede zwischen Forschenden und Praxisakteuren nicht zu verwischen.

- Hinweise darauf, worüber während der Umsetzung Reflexions-Bedarf besteht und welche Instrumentalisierungsrisiken es zu vermeiden gilt.

Beziehung der Praxisakteure untereinander - wie mit Macht und Ausschluss umgehen?

- Ausführungen dazu, welche Beziehung zwischen den Praxisakteuren sowie zwischen den Praxisakteuren und dem Praxisfeld die Methode voraussetzt und wie sie diese Beziehungen verändert.

- Hinweis darauf, welche Beziehungen zwischen den Praxisakteuren sowie zwischen den Praxisakteuren und dem Praxisfeld die Umsetzung der Methode gefährden könnten bzw. welche die Umsetzung der Methode verunmöglichen. Ausführungen dazu, wo sich Machtstrukturen (innerhalb wie außerhalb des Projekts) verzerrend auf die Umsetzung der Methode auswirken können und wie dem entgegengewirkt werden kann. Hinweis auf soziale Formen des Ausschlusses, die für die Methode relevant sind, und wie diesen zu begegnen ist. Hinweise darauf, wo bei der Umsetzung der Methode die Gefahr aufkommen könnte, dass die Praxisakteure sich nicht ausreichend von ihrem Praxisfeld und ihrer Rolle darin ,distanzieren' könnten, und wie sich diese Gefahr verringern lässt.

- Hinweise darauf, worüber während der Umsetzung Reflexions-Bedarf besteht.

\section{Dokumentation - wie Transparenz herstellen?}

- Ausführungen dazu, welche Entscheidungen bei der Vorbereitung, Umsetzung oder Nachbereitung der Methode dokumentiert werden müssen und wer über welche Entscheidungen informiert werden muss, d. h. worüber Transparenz hergestellt werden muss mit Blick auf die Nachvollziehbarkeit.

- Hinweise darauf, welche Daten und (Zwischen-)Ergebnisse dokumentiert werden müssen sowie welche Dokumentation den Forschenden und welche den Praxisakteuren zur Verfügung gestellt werden muss. Hinweise auf Vorkehrungen, die mit Blick auf den Schutz der Beteiligten und/oder auf den Schutz geistigen Eigentums zu treffen sind. Falls möglich und angebracht: Ausführungen dazu, wie die Dokumentation technisch erfolgen könnte.

- Falls erforderlich: Ausführungen darüber, wie über das Vorgehen und die Ergebnisse gegen außen berichtet werden sollte. 
Der Blick auf die Reflexion der Erfahrungen

Aufnahme der Methode - wie kommt sie an?

- Ausführungen dazu, wie Praxisakteure die Methode wahrgenommen und aufgenommen haben, d. h. was bei welchen Praxisakteuren besonders auf Anklang stieß, womit welche Praxisakteure ggf. Mühe bekundeten, welche Handlungen welchen Praxisakteuren leicht bzw. schwergefallen sind. Hinweise darauf, welche Konsequenzen sich daraus ergeben für die Wahl und/oder für die (Anpassung und) Umsetzung der Methode.

- Hinweise darauf, welche Instrumente verwendet werden könnten und auf welche Indizien geachtet werden sollte, um in der Umsetzung der Methode feststellen zu können, wie die Praxisakteure auf die Methode reagieren.

\section{Angemessenheit - welche Erwartungen, welche mögliche Schäden?}

- Ausführungen dazu, was die Methode bei den Praxisakteuren bzw. im Praxisfeld auslösen kann, d. h. welche Erwartungen durch die Methode geweckt werden können. Hinweise darauf, welchen dieser Erwartungen zu entsprechen ist und welchen nicht entsprochen werden muss bzw. welchen nicht entsprochen werden kann.

- Hinweise darauf, welches ,Schäden' sind (gemessen an welchen Kriterien), die durch die Methode verursacht werden können, und Hinweise darauf, wie diese vermieden werden können.

- Hinweise darauf, ob es Praxisfelder bzw. Konstellationen von Praxisakteuren gibt, die eine Umsetzung der Methode verunmöglichen oder mindestens nicht ratsam scheinen lassen.

\section{Nutzen und Anwendung der vorgeschlagenen Gütekriterien}

Drei Gründe sprechen dafür, sich mit wissenschaftlichen Gütekriterien rund um Methoden der partizipativen Wissenserzeugung in der transdisziplinären Forschung zu befassen: Erstens die Zweifel an der Gültigkeit partizipativ erzeugten Wissens innerhalb der Wissenschaft. Zweitens das Fehlen methodischer Gütekriterien dafür bzw. die fehlende Diskussion darüber, wie die Nachvollziehbarkeit, und damit die Glaubwürdigkeit und die prinzipielle Kritisierbarkeit partizipativ erzeugter Ergebnisse hergestellt werden kann und soll. Drittens der forschungspraktische Bedarf nach einem umfangreichen Pool an bewährten Methoden in der Praxis der (inter- und) transdisziplinären Forschung außerhalb wie innerhalb der Reallaborforschung. 
Der hier vorgestellte Katalog an Gütekriterien für Methoden der partizipativen Wissenserzeugung in der transdisziplinären Forschung soll eine Antwort sein auf diese Defizite. Der Beitrag zur Methodik, der mit diesem Katalog geleistet werden soll, ist somit ein methodologischer, vor allem aber auch ein forschungspraktischer. Dabei wird nicht beansprucht, neu und originell zu sein. Vielmehr wird der Blick auf die im Kontext partizipativer Forschung, Aktionsforschung, Handlungsforschung oder Interventionsforschung bereits seit längerem geführte Debatte gelenkt, verbunden mit der Aufforderung, sich an die im Rahmen dieser Debatte entwickelten Kriterien zu erinnern und sich dieser zu bedienen.

Die vorgestellten Gütekriterien sollten, so der Vorschlag, bei der Wahl (inkl. Entwicklung und Kombination) und bei der Umsetzung von Methoden der partizipativen Wissenserzeugung sowie bei der Dokumentation und Reflexion des methodischen Vorgehens berücksichtigt werden. Bei der Erläuterung der vorgeschlagenen Kriterien wurde die Perspektive der Aufbereitung von Methoden eingenommen (die sich, das dürfte evident sein, an denselben Kriterien orientieren sollte). Aus diesen Erläuterungen lassen sich leicht die Punkte ableiten, auf die z. B. bei der Dokumentation des Vorgehens bei der partizipativen Wissenserzeugung in einem transdisziplinären Projekt eingegangen werden sollte, oder die Fragen, die dazu dienen können, das methodische Vorgehen in einem Forschungsprojekt zu reflektieren. In welchen Kontexten sich die vorgeschlagenen Gütekriterien bewähren und wie sie weiterzuentwickeln sind, wird sich im methodologischen Diskurs und in den forschungspraktischen Erfahrungen entscheiden müssen.

\section{Dank}

Die Autorin und der Autor danken für die Diskussionen über den KriterienVorschlag bzw. frühere Fassungen des Textes Marius Albiez, Sophia Alcántara, Thomas Becker, Carolin Becker-Leifhold, Richard Beecroft, Matthias Bergmann, Michael Bossert, Marius Gantert, Ulrike Gerhard, Michael Kalff, Jan Lutz, Editha Marquardt, Aida Nejad, Oliver Parodi, Felix M. Piontek, Michael Pregernig, Regina Rhodius, Ines-Ulrike Rudolph, Holger Schallehn, Andreas Seebacher, Antje Stokman, Colette Waitz und Christina West. Insbesondere möchten sie auch den zwei externen Gutachter(inne)n für die konkreten Hinweise zur Verbesserung des Textes danken. 


\section{Literatur}

Altrichter, H., \& Feindt, A. (2008). Handlungs- und Praxisforschung. In W. Helsper \& J. Böhme (Hrsg.), Handbuch der Schulforschung (S. 449-466). Wiesbaden: Springer VS.

Arnold, A., \& Piontek, F. M. (2018). Zentrale Begriffe im Kontext der Reallaborforschung. In R. Defila \& A. Di Giulio (Hrsg.), Transdisziplinär und transformativ forschen. Eine Methodensammlung (S. 143-154). Wiesbaden: Springer VS.

Baur, N., \& Blasius, J. (Hrsg.). (2014a). Handbuch Methoden der empirischen Sozialforschung. Wiesbaden: Springer VS.

Baur, N., \& Blasius, J. (2014b). Methoden der empirischen Sozialforschung. Ein Überblick. In N. Baur \& J. Blasius (Hrsg.), Handbuch Methoden der empirischen Sozialforschung (S. 41-62). Wiesbaden: Springer VS.

Beecroft, R., Trenks, H., Rhodius, R., Benighaus, C., \& Parodi, O. (2018). Reallabore als Rahmen transformativer und transdisziplinärer Forschung: Ziele und Designprinzipien. In R. Defila \& A. Di Giulio (Hrsg.), Transdisziplinär und transformativ forschen. Eine Methodensammlung (S. 75-100). Wiesbaden: Springer VS.

Bergmann, M., Jahn, T., Knobloch, T., Krohn, W., Pohl, C., \& Schramm, E. (2010). Methoden transdisziplinärer Forschung. Ein Überblick mit Anwendungsbeispielen. Frankfurt a. M., New York: Campus.

Bergold, J., \& Thomas, S. (2010). Partizipative Forschung. In G. Mey \& K. Mruck (Hrsg.), Handbuch Qualitative Forschung in der Psychologie (S. 333-344). Wiesbaden: Springer VS.

BGL-Themenheft (2017). Reallabore als Forschungsformat nachhaltiger Stadtentwicklung. Berichte. Geographie und Landeskunde, 91 (1). (im Druck).

Bogner, A., Littig, B., \& Menz, W. (2014). Interviews mit Experten. Eine praxisorientierte Einführung. Wiesbaden: Springer VS.

Böhme, J. (2008). Qualitative Schulforschung auf Konsolidierungskurs: interdisziplinäre Spannungen und Herausforderungen. In W. Helsper \& J. Böhme (Hrsg.), Handbuch der Schulforschung (S. 125-155). Wiesbaden: Springer VS.

Bonn, A., Richter, A., Vohland, K., Pettibone, L., Brandt, M., Feldmann, R., Goebel, C., Grefe, C., Hecker, S., Hennen, L., Hofer, H., Kiefer, S., Klotz, S., Kluttig, T., Krause, J., Küsel, K., Liedtke, C., Mahla, A., Neumeier, V., Premke-Kraus, M., Rillig, M. C., Röller, O., Schäffler, L., Schmalzbauer, B., Schneidewind, U., Schumann, A., Settele, J., Tochtermann, K., Tockner, K., Vogel, J., Volkmann, W., von Unger, H., Walter, D., Weisskopf, M., Wirth, C., Witt, T., Wolst, D., \& Ziegler, D. (2016). Grünbuch Citizen Science Strategie 2020 für Deutschland. Helmholtz-Zentrum für Umweltforschung, Deutsches Zentrum für integrative Biodiversitätsforschung Halle-Jena-Leipzig, Leipzig, Museum für Naturkunde Berlin, Leibniz-Institut für Evolutions- und Biodiversitätsforschung, Berlin-Brandenburgisches Institut für Biodiversitätsforschung, Berlin. 
Breuer, F. (2010). Wissenschaftstheoretische Grundlagen qualitativer Methodik in der Psychologie. In G. Mey \& K. Mruck (Hrsg.), Handbuch Qualitative Forschung in der Psychologie (S. 35-49). Wiesbaden: Springer VS.

CASS \& ProClim- (1997). Forschung zu Nachhaltigkeit und Globalem Wandel - Wissenschaftspolitische Visionen der Schweizer Forschenden. Bern: ProClim-/SANW. https://naturwissenschaften.ch/service/publications/75640-visionen-der-forschenden. Zugegriffen am 21.01.2018.

Collins, H. M., \& Evans, R. (2002). The third wave of science studies: Studies of expertise and experience. Social Studies of Science, 32 (2), (S. 235-296).

Cuhls, K. (2015). Ziele und Rahmenbedingungen. In L. Gerhold, D. Holtmannspötter, C. Neuhaus, E. Schüll, B. Schulz-Montag, K. Steinmüller \& A. Zweck (Hrsg.), Standards und Gütekriterien der Zukunftsforschung. Ein Handbuch für Wissenschaft und Praxis (S. 86-93). Wiesbaden: Springer VS.

Defila, R., \& Di Giulio, A. (2018). Eine Reflexion über Legitimation, Partizipation und Intervention im Kontext transdisziplinärer Forschung. In M. Ukowitz \& R. Hübner (Hrsg.), Partizipation und Intervention. Wege der Vermittlung in der transdisziplinären Forschung. Interventionsforschung, Bd. 3. Wiesbaden: Springer VS. (im Druck).

Defila, R., \& Di Giulio, A. (2015). Integrating knowledge: challenges raised by the „Inventory of Synthesis“. Futures, 65, Special Issue Transdisciplinarity revisited, (S. 123135). doi:10.1016/j.futures.2014.10.013, online verfügbar seit 05.11.2014.

Defila, R., \& Di Giulio, A. (1998). Interdisziplinarität und Disziplinarität. In J.-H. Olbertz (Hrsg.), Zwischen den Fächern - über den Dingen? Universalisierung versus Spezialisierung akademischer Bildung (S. 111-137). Opladen: Leske \& Budrich. doi: 10.1007/ 978-3-322-90935-0_6.

Defila, R., Di Giulio, A., \& Scheuermann, M. (2008). Management von Forschungsverbünden - Möglichkeiten der Professionalisierung und Unterstützung. Herausgegeben von der Deutschen Forschungsgemeinschaft (DFG). Weinheim: Wiley-VCH. http://www.dfg.de/download/pdf/dfg_im_profil/geschaeftsstelle/publikationen/manage ment_forschungsverbuende.pdf. Zugegriffen am 25.01.2018.

Defila, R., Di Giulio, A., \& Scheuermann, M. (2006). Forschungsverbundmanagement. Handbuch für die Gestaltung inter- und transdisziplinärer Projekte. Zürich: vdf Hochschulverlag an der ETH Zürich.

Di Giulio, A., Defila, R., \& Brückmann, Th. (2016). „Das ist halt das eine ... Praxis, das andere ist Theorie" - Prinzipien transdisziplinärer Zusammenarbeit im Forschungsalltag. In R. Defila \& A. Di Giulio (Hrsg.), Transdisziplinär forschen - zwischen Ideal und gelebter Praxis. Hotspots, Geschichten, Wirkungen (S. 189-286). Frankfurt a. M., New York: Campus.

Eckart, J., Ley, A., Häußler, E., \& Erl, Th. (2018). Leitfragen für die Gestaltung von Partizipationsprozessen in Reallaboren. In R. Defila \& A. Di Giulio (Hrsg.), Transdisziplinär und transformativ forschen. Eine Methodensammlung (S. 105-135). Wiesbaden: Springer VS. 
Flick, U. (2010). Gütekriterien qualitativer Forschung. In G. Mey \& K. Mruck (Hrsg.), Handbuch Qualitative Forschung in der Psychologie (S. 395-407). Wiesbaden: Springer VS.

Flick, U., von Kardorff, E., \& Steinke, I. (Hrsg.) (2000a). Qualitative Forschung - Ein Handbuch. Hamburg: Rowohlts Enzyklopädie.

Flick, U., von Kardorff, E., \& Steinke, I. (2000b). Was ist qualitative Forschung? Einleitung und Überblick. In U. Flick, E. von Kardorff \& I. Steinke (Hrsg.), Qualitative Forschung - Ein Handbuch (S. 13-29). Hamburg: Rowohlts Enzyklopädie.

GAIA Special Issue (2018). Labs in the real world: Advancing Transdisciplinarity and Transformations. GAIA, 27 (S1). (im Druck).

Gerhold, L. (2015). Methodenwahl und Methodenkombination. In L. Gerhold, D. Holtmannspötter, C. Neuhaus, E. Schüll, B. Schulz-Montag, K. Steinmüller \& A. Zweck (Hrsg.), Standards und Gütekriterien der Zukunftsforschung. Ein Handbuch für Wissenschaft und Praxis (S. 111-120). Wiesbaden: Springer VS.

Gerhold, L., Holtmannspötter, D., Neuhaus, C., Schüll, E., Schulz-Montag, B., Steinmüller, K., \& Zweck, A. (Hrsg.) (2015). Standards und Gütekriterien der Zukunftsforschung. Ein Handbuch für Wissenschaft und Praxis. Wiesbaden: Springer VS.

Gutscher, H., Hirsch, G., \& Werner, K. (1996). Vom Sinn der Methodenvielfalt in den Sozial- und Geisteswissenschaften. In R. Kaufmann-Hayoz \& A. Di Giulio (Hrsg.), Umweltproblem Mensch. Humanwissenschaftliche Zugänge zu umweltverantwortlichem Handeln (S. 43-78). Bern, Stuttgart, Wien: Verlag Paul Haupt.

Helsper, W., \& Böhme, J. (Hrsg.) (2008). Handbuch der Schulforschung. Wiesbaden: Springer VS.

Hussy, W., Schreier, M., \& Echterhoff, G. (Hrsg.) (2013). Forschungsmethoden in Psychologie und Sozialwissenschaften für Bachelor. Berlin, Heidelberg: Springer.

Klein, J. T. (2014). Interdisciplinarity and Transdisciplinarity: Keyword Meanings for Collaboration Science and Translational Medicine. Journal of Translational Medicine \& Epidemiology, 2 (2), (S. 1024-30).

Klein, J. T. (2010). A taxonomy of interdisciplinarity. In R. Frodeman, J. T. Klein \& C. Mitcham (Hrsg.), The Oxford handbook of interdisciplinarity (S. 15-30). Oxford: Oxford University Press.

Krainer, L., \& Lerchster, R. E. (Hrsg.) (2012a). Interventionsforschung. Bd. 1. Paradigmen, Methoden, Reflexionen. Wiesbaden: Springer VS.

Krainer, L., \& Lerchster, R. E. (2012b). Interventionsforschung: Paradigmen, Methoden, Reflexionen. In L. Krainer \& R. E. Lerchster (Hrsg.), Interventionsforschung. Bd. 1. Paradigmen, Methoden, Reflexionen (S. 9-19). Wiesbaden: Springer VS.

Krainer, L., Lerchster, R., \& Goldmann, H. (2012). Interventionsforschung in der Praxis. In L. Krainer \& R. E. Lerchster (Hrsg.), Interventionsforschung. Bd. 1. Paradigmen, Methoden, Reflexionen (S. 175-243). Wiesbaden: Springer VS. 
Krohn, W., Grunwald, A., \& Ukowitz, M. (2017). Transdisziplinäre Forschung revisited. Erkenntnisinteresse, Forschungsgegenstände, Wissensform und Methodologie. GAIA 26 (4), (S. 341-347). doi:10.14512/gaia.26.4.11.

Lerchster, R. (2012). Zentrale Grundannahmen der Interventionsforschung. In L. Krainer \& R. E. Lerchster (Hrsg.), Interventionsforschung. Bd. 1. Paradigmen, Methoden, Reflexionen (S. 23-73). Wiesbaden: Springer VS.

Loibl, M. C. (2005). Spannungen in Forschungsteams. Hintergründe und Methoden zum konstruktiven Abbau von Konflikten in inter- und transdisziplinären Projekten. Heidelberg: Carl-Auer-Systeme.

Mayring, P. (2010). Design. In G. Mey \& K. Mruck (Hrsg.), Handbuch Qualitative Forschung in der Psychologie (S. 225-237). Wiesbaden: Springer VS.

McDonald, D., Bammer, G., \& Dean, P. (2009). Dialogue tools for research integration. Canberra, Australia: ANU E Press, The Australian National University.

Mey, G., \& Mruck, K. (Hrsg.) (2010). Handbuch Qualitative Forschung in der Psychologie. Wiesbaden: Springer VS.

Mobjörk, M. (2010). Consulting versus participatory transdisciplinarity: A refined classification of transdisciplinary research. Futures, 42 (8), (S. 866-873). doi: 10.1016/ j.futures.2010.03.003.

Niederberger, M., \& Wassermann, S. (Hrsg.) (2015). Methoden der Experten- und Stakeholdereinbindung in der sozialwissenschatlichen Forschung. Wiesbaden: Springer VS.

Nolting, K., Illge, L., Bottin, K., Schäfer, M., \& Wemheuer, C. (2008). Kooperieren - aber wie? Ein Leitfaden zum Aufbau von Kooperationsbeziehungen zwischen LokalenAgenda-21-Initiativen und Akteuren aus Wirtschaft und Wissenschaft. Berlin: Institut für Zukunftsstudien; Zentrum Technik und Gesellschaft.

Pettibone, L., Blättel-Mink, B., Balázs, B., Di Giulio, A., Göbel, C., Heubach, K., Hummel, D., Lundershausen, J., Lux, A., Potthast, T., Vohland K., \& Wyborn, C. (2018). Transdisciplinary Sustainability Research and Citizen Science: Options for Mutual Learning. GAIA. (in review).

Pohl, C., \& Hirsch Hadorn, G. (2006). Gestaltungsprinzipien für die transdisziplinäre Forschung. Ein Beitrag des td-net. München: oekom.

Potthast, Th. (2015). Ethics in the Sciences beyond Hume, Moore and Weber - Taking Epistemic-Moral Hybrids Seriously. In S. Meisch, J. Ludershausen, L. Bossert \& M. Rockoff (Hrsg.), Ethics of science in the research for sustainable development (S. 131-153). Baden-Baden: Nomos.

Prengel, A., Heinzel, F., \& Carle, U. (2008). Methoden der Handlungs-, Praxis- und Evaluationsforschung. In W. Helsper \& J. Böhme (Hrsg.), Handbuch der Schulforschung (S. 181-197). Wiesbaden: Springer VS.

Przyborski, A., \& Wohlrab-Sahr, M. (2014). Forschungsdesigns für die qualitative Sozialforschung. In N. Baur \& J. Blasius (Hrsg.), Handbuch Methoden der empirischen Sozialforschung (S. 117-133). Wiesbaden: Springer VS. 
Quint, A., Alcántara, S., \& Seebacher, A. (2018). Der Partizipationsmythos „Partizipation in Reallaboren ist per se transparent und muss es auch sein“. In R. Defila \& A. Di Giulio (Hrsg.), Transdisziplinär und transformativ forschen. Eine Methodensammlung (S. 69-73). Wiesbaden: Springer VS.

Rabelt, V., Büttner, T., \& Simon, K.-H. (Hrsg.) (2007). Neue Wege in der Forschungspraxis. Begleitinstrumente in der transdisziplinären Nachhaltigkeitsforschung. München: oekom.

Schäpke, N., Stelzer, F., Bergmann, M., Singer-Brodowski, M., Wanner, M., Caniglia G., \& Lang, D. (2017). Reallabore im Kontext transformativer Forschung. Ansatzpunkte zur Konzeption und Einbettung in den internationalen Forschungsstand. (No. 1/2017). Leuphana Universität Lüneburg, Institut für Ethik und Transdisziplinäre Nachhaltigkeitsforschung.

Scheele, B., \& Groeben, N. (2010). Dialog-Konsens-Methoden. In G. Mey \& K. Mruck (Hrsg.), Handbuch Qualitative Forschung in der Psychologie (S. 506-523). Wiesbaden: Springer VS.

Schneidewind, U., \& Singer-Brodowski, M. (2015). Vom experimentellen Lernen zum transformativen Experimentieren. Reallabore als Katalysator für eine lernende Gesellschaft auf dem Weg zu einer Nachhaltigen Entwicklung. Zeitschrift für Wirtschaftsund Unternehmensethik, 16 (1), (S. 10-23).

Schophaus, M., Schön, S., \& Dienel, H.-L. (Hrsg.) (2004). Transdisziplinäres Kooperationsmanagement. Neue Wege in der Zusammenarbeit zwischen Wissenschaft und Gesellschaft. München: oekom.

Schreier, M. (2013). Qualitative Forschungsmethoden. In W. Hussy, M. Schreier \& G. Echterhoff (Hrsg.), Forschungsmethoden in Psychologie und Sozialwissenschaften für Bachelor (S. 189-221). Berlin, Heidelberg: Springer VS.

Schüll, E., \& Gerhold, L. (2015): Nachvollziehbarkeit. In L. Gerhold, D. Holtmannspötter, C. Neuhaus, E. Schüll, B. Schulz-Montag, K. Steinmüller \& A. Zweck (Hrsg.), Standards und Gütekriterien der Zukunftsforschung. Ein Handbuch für Wissenschaft und Praxis (S. 94-99). Wiesbaden: Springer VS.

Seel, N. M., \& Hanke, U. (Hrsg.) (2015a). Erziehungswissenschaft. Lehrbuch für Bachelor-, Master- und Lehramtsstudierende. Berlin, Heidelberg: Springer VS.

Seel, N. M., \& Hanke, U. (2015b). Methodik der Erziehungswissenschaft. In N. M. Seel \& U. Hanke (Hrsg.), Erziehungswissenschaft. Lehrbuch für Bachelor-, Master- und Lehramtsstudierende (S. 769-852). Berlin, Heidelberg: Springer VS.

Steinke, I. (1999). Kriterien qualitativer Forschung. Ansätze zur Bewertung qualitativempirischer Sozialforschung. Weinheim, München: Juventa.

TATuP-Schwerpunkt (2016). Reallabore als Orte der Nachhaltigkeitsforschung und Transformation. Technikfolgenabschätzung - Theorie und Praxis, 25 (3), (S. 4-51).

Ukowitz, M. (2012). Interventionsforschung im Kontext transdisziplinärer Wissenschaften. In L. Krainer \& R. E. Lerchster (Hrsg.), Interventionsforschung. Bd. 1. Paradigmen, Methoden, Reflexionen (S. 75-101). Wiesbaden: Springer VS. 
von Blanckenburg, C., Böhm, B., Dienel, H.-L., \& Legewie, H. (2005). Leitfaden für interdisziplinäre Forschergruppen: Projekte initiieren - Zusammenarbeit gestalten. Stuttgart: Franz Steiner.

von Unger, H. (2014). Partizipative Forschung. Einführung in die Forschungspraxis. Wiesbaden: Springer VS.

Wagner, F., \& Grunwald, A. (2015). Reallabore als Forschungs- und Transformationsinstrument. Die Quadratur des hermeneutischen Zirkels. GAIA, 24 (1), (S. 26-31).

\section{Online-Methodensammlungen}

About Interdisciplinarity - http://sites.google.com/a/ualberta.ca/rick-szostak

Integration and Implementation Sciences - http://i2s.anu.edu.au/resources

Short Guides to Interdisciplinarity - https://www.tinyurl.com/idwiki

td-net's toolbox - http://www.naturalsciences.ch/topics/co-producing_knowledge

Team Science Toolkit - https://www.teamsciencetoolkit.cancer.gov

(auf alle zugegriffen am 21.01.2018).

Open Access Dieses Kapitel wird unter der Creative Commons Namensnennung 4.0 International Lizenz (http://creativecommons.org/licenses/by/4.0/deed.de) veröffentlicht, welche die Nutzung, Vervielfältigung, Bearbeitung, Verbreitung und Wiedergabe in jeglichem Medium und Format erlaubt, sofern Sie den/die ursprünglichen Autor(en) und die Quelle ordnungsgemäß nennen, einen Link zur Creative Commons Lizenz beifügen und angeben, ob Änderungen vorgenommen wurden.

Die in diesem Kapitel enthaltenen Bilder und sonstiges Drittmaterial unterliegen ebenfalls der genannten Creative Commons Lizenz, sofern sich aus der Abbildungslegende nichts anderes ergibt. Sofern das betreffende Material nicht unter der genannten Creative Commons Lizenz steht und die betreffende Handlung nicht nach gesetzlichen Vorschriften erlaubt ist, ist für die oben aufgeführten Weiterverwendungen des Materials die Einwilligung des jeweiligen Rechteinhabers einzuholen. 